\section{The problem with an epidemiological index for dental erosion}

\author{
A. Milosevic ${ }^{1}$
}

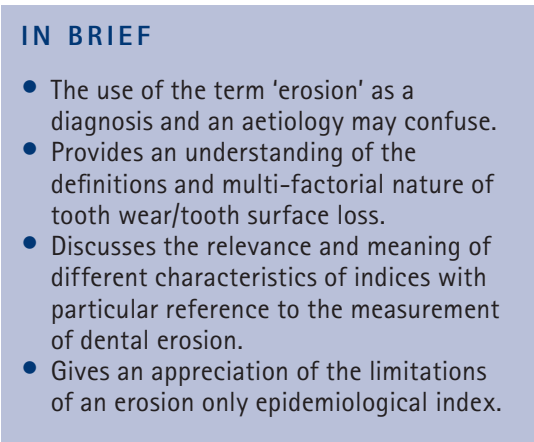

This article reviews and discusses the difficulties posed by the application of epidemiological indices for the measurement of dental erosion. It covers several aspects of index design.

\section{INTRODUCTION}

It is generally accepted that wear is age related and therefore a physiological process. The ageing dentate population will inevitably have some degree of tooth wear. Wear is a physical process occurring whenever two or more surfaces rub against each other. The physical science related to the study of wear, friction and lubrication is tribology. The tribological term for dental attrition (direct tooth to tooth contact wear) occurring during bruxist activity is termed 2-body abrasion. ${ }^{1}$ Dental abrasion may also be 2-body wear, occurring when a non-tooth surface rubs against the tooth such as a nail or smoker's pipe. Tooth brushing, however, is likely to be 3-body wear as there is an intervening slurry of toothpaste between the brush and the tooth. These wear mechanisms are accelerated in the presence of acids, which demineralise and soften the surface.

\section{TERMINOLOGY, AETIOLOGY AND SITE SPECIFICITY}

In certain cases abrasion, attrition and/or erosion result in unacceptable or pathological levels of tooth surface loss. This latter term, synonymous with the term tooth wear, encompasses the multi-factorial combination of these three processes commonly present in any one case.

'Department of Restorative Dentistry, Liverpool University Dental Hospital, Pembroke Place, Liverpool, L3 5PS

Correspondence to: Dr Alexander Milosevic

Email: alexander.milosevic@rlbuht.nhs.uk

\section{Refereed Paper}

Accepted 27 July 2011

DOI: 10.1038/sj.bdj.2011.722

${ }^{\circledR}$ British Dental Journal 2011; 211: 201-203
Furthermore, aetiology is not presumed. At the individual patient level this can confront the clinician with a diagnostic difficulty when trying to identify what aetiological risk factors are present. Questioning at the chairside will hopefully identify the risk factors and facilitate appropriate intervention. The epidemiologist, however, does not have the luxury of asking subjects about risk factors in a prevalence study although questionnaires regarding risks are used when determining associations. The first potential problem with an erosion index used in prevalence studies is the assumption of aetiology. Erosion is used as both the descriptor of the worn surface and the aetiology. Indeed, the terms abrasion, attrition and erosion are aetiologies. Figure 1 illustrates the problem. This 43-year-old male from Hong Kong chewed chicken bones but the intake of dietary acid was low. The initial presentation is suggestive of dental erosion but abrasion was the predominant risk factor.

The erosion index must only measure tooth surface loss as a result of acid dissolution and exclude any change consequential to attrition and abrasion. Diagnostic criteria for early erosion of enamel include loss of mammelons or perikymata. These anatomic features can also be worn away by attrition and abrasion. Some researchers have tried to overcome this problem by selective data analysis, for example, by excluding incisal edges and occlusal surfaces and assessing only facial/palatal surfaces. The effect, however, from direct tooth-to-tooth guidance during protrusion and tongue abrasion cannot be ruled out when assessing palatal erosion. ${ }^{2}$
Similarly, labial and buccal surfaces are unlikely to undergo attrition in normal class I relationships but in clinical situations of deep overbite the labial surfaces of lower incisors do wear and conversely with a reverse overjet upper labial wear can occur. It is therefore difficult to measure erosion solely on the basis of site specificity.

\section{MEASUREMENT VALIDITY}

From the above discussion it should be apparent that measurement validity for a purely erosion index is doubtful. Measurement validity is an expression of the degree to which a measure actually measures what it purports to measure. ${ }^{3}$ An erosion only index may also measure tooth surface change as a result of abrasion and attrition or possibly a fractured surface such as an incisal edge. Erosion indices are by their nature nonspecific despite attempts by various workers to develop stringent diagnostic criteria.

\section{SPECIFICITY VS SENSITIVITY}

A specific test has few false positives whereas sensitivity detects a high proportion of true cases and thus reduces the number of false negatives. If diagnostic criteria are strict there will be few false positives but the test or index will be insensitive. A balance needs to be achieved between sensitivity and specificity. A specific test, however, is generally preferred, even if some sensitivity is lost. This improves predictive value which is important when screening (see later). Furthermore, a reference test is the standard against which an index is compared. 
There is no reference test for erosion although the clinical or visual identification of exposed dentine on worn occlusal/ incisal surfaces had only fair agreement when compared to the histologically sectioned teeth after clinical examination. ${ }^{4}$ The diagnosis of exposed dentine was concluded to be difficult although highly specific (0.88). Importantly in the histological sections dentine was exposed in all cases of cupping and grooving even if only minor substance loss occurred. It would appear that training and calibration of examiners is critical for erosion and tooth wear studies. This sentiment was echoed in the 1993 UK National Child Dental Health Survey when erosion was assessed for the first time and low levels of agreement between dentists were noted for enamel erosion only. ${ }^{5}$ Despite these difficulties, dichotomising on the presence or absence of dentine in any index seems appropriate and this is the basis for the modified Tooth Wear Index. ${ }^{6}$ However, this once again is not an erosion index. Given the difficulty with an erosion specific index, the measurement of tooth wear is more appropriate and should have good sensitivity although it must be recognised that measurement of erosion is not the same as measurement of tooth wear.

\section{SCREENING VS MONITORING}

Screening is not intended to be diagnostic but is a rapid examination or test aimed at detecting disease not under medical care. It may identify risk factors, genetic tendency or early disease and disorder. Different types of screening exist: mass, multiple and prescriptive. ${ }^{3}$ Mass screening of the whole population for a dental problem is inappropriate although predictive screening aims to detect the early stage of disease. Monitoring is the episodic and sequential measurement of changes in health status typically before and after an intervention. The purpose of screening is to identify unrecognised disease while monitoring assesses the effect of intervention. Both should be continuous. One problem with screening is that the subjects most at risk do not present for screening and monitoring depends upon precision of the test or measure. The Basic Periodontal Examination (BPE) is an example of a screening tool reliant on periodontal probing which has well known limitations of accuracy. The recent BEWE or Basic Erosive Wear Examination has been described as a screening and monitoring system for tooth wear although the introduction of 'erosive wear' which implies erosion may serve to confuse dentists. ${ }^{7,8}$ It has a four point grading scale from 0 to 3 with scores 2 and 3 having diagnostic criteria of hard tissue loss of $<50 \%$ or $>50 \%$ of the surface respectively. Like the BPE it is sextant based and not quadrant based as previously described. ${ }^{8}$ Only the highest score for one surface is recorded for each sextant. The six scores are then summed to give an overall score for the mouth with a maximal score of 18 indicative of the worst possible case. 'Risk' or 'complexity levels' guide clinical management with the high risk category of 14+ requiring oral hygiene, dietary assessment, identification of aetiological factors, fluoridation and other strategies, monitoring by various means, avoidance of restorations ideally but in cases of severe progression consider special care that may involve restorations. The management strategies are the same for medium risk scores, between 9 and 13, except for the exclusion of special care that may involve restorations. It is not clear what is meant by special care as complex treatment is not always necessary and restoration by addition of dental composite has been successful. Such treatment can be carried out in the primary care setting. There is no inclusion of pain, sensitivity or the common presenting complaint of poor aesthetics which often drive treatment. Arguably, the BEWE is attempting to do several things: monitor; screen; index of treatment need; measure tooth wear and/or erosion.

The BEWE has been advocated for the monitoring of individual cases but later it has been stated that "the intention of the BEWE is not to measure progression as the distinction between the various levels is crude.' ${ }^{8}$ There is a lack of clarity regarding the BEWE which is disappointing given the need for a validated and widely accepted index to measure tooth wear and erosion.

\section{MARKER/INDEX TEETH VS FULL MOUTH EXAMINATION}

Prevalence studies in adolescent children indicate that commonly worn teeth are the incisors, canines and first molars. ${ }^{9-11}$ Whether important information is not
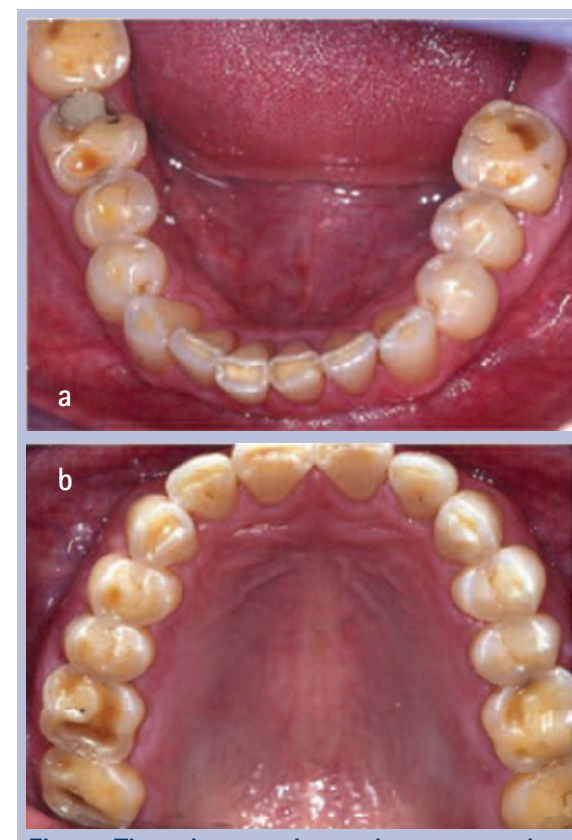

Fig. 1 These images shows the upper and lower teeth in a middle aged male who chewed bones, especially chicken bones, over a lifetime. Note that the upper palatal surfaces appear intact and that the wear is mainly confined to the incisal edges, canine tips and occlusal surfaces. The occlusal surfaces are cupped which may draw the clinician to diagnose acid erosion but dietary acid intake was very low

captured if only certain teeth and sites are assessed in an index is open to debate. Several different partial recording systems were compared to determine which teeth would provide good sensitivity in dentate older adults in England. ${ }^{12}$ The data when scoring the 12 anterior teeth related closely to the results when scoring the whole mouth. The modified Tooth Wear Index examines the 12 anterior teeth and the four first permanent molars with little loss of sensitivity. Indices for erosion may similarly have index teeth but whether the teeth and sites should be the same as previously advocated for tooth wear is not determined. The 2003 UK National Child Dental Health survey recorded tooth surface loss on the labial and palatal surfaces of the upper incisors and the occlusal surfaces of the first molars, the latter surfaces being incorporated since the 1993 survey. ${ }^{13}$

\section{DEPTH VS AREA}

Enamel surface loss is difficult to see clinically in the dental surgery but more so in a field setting without adequate light and drying facilities. Loss of enamel surface characteristics such as mammelons and 
perikymata have been used as diagnostic criteria in the Tooth Wear Index which is appropriate as it is likely that wear has resulted in loss of these anatomical features. ${ }^{14}$ Loss of smooth surface enamel is more difficult to see, although if fairly advanced ridges and pseudo chamfer margins are detectable within the enamel. Inter-examiner reproducibility for measuring enamel lesions may be low and furthermore there can be no certainty that enamel lesions are solely caused by acid erosion. Relatively low levels of agreement between examiners in identification of enamel tooth surface loss was noted in the 2003 UK National Child Dental Health survey. ${ }^{13}$

Many indices describe area and depth of surface loss in terms of enamel and dentine involvement. The amount of eroded dentine expressed in percentage terms of the area of the surface was first described by Eccles who used $<1 / 3$ or $>1 / 3$ as the threshold for the grades Class II or Class III. ${ }^{15} \mathrm{~A}$ better descriptor is exposed dentine as this is exactly what is seen. Dentinal exposure should not, however, be a surrogate marker for erosion or any other mode of wear.

Greater concordance between examiners may be possible once dentine is exposed. ${ }^{13}$ The proportion of exposed dentine on any given surface is typically stated as less than or more than a third or in some indices a $50 \%$ threshold is applied. The BEWE has a $50 \%$ threshold for the area of hard tissue loss of the surface irrespective of whether this is enamel or dentine. Cut-off points are arbitrary but any threshold should be realistic. For instance, if epidemiological studies find that there are only a few or no cases of dentinal exposure above $50 \%$ on occlusal surfaces of first molars in adults, then it may be better to accept a lower threshold to capture more cases in the category of greater severity.

\section{IN CONCLUSION}

Dental erosion is of interest to academics and clinicians. Its prevalence, incidence, severity and associated risks are important to know in order to plan appropriate prevention and treatment both at the individual and the public health level. The epidemiological measures thus far are recognised as flawed and that a new epidemiological tool needs to be developed. ${ }^{16}$ Unfortunately, there is still widespread confusion and misunderstanding in the terminology partly because European researchers prefer the term erosion for non-cariogenic changes. ${ }^{8}$ The focus on erosion at the expense of attrition has driven researchers to develop indices or measures putatively specific for erosion despite the wide acceptance of the multi-factorial nature of the tooth wear process. Attrition is still a common problem. Recognition of these issues has led to a plethora of indices both old and new being used in research but with limited ability to compare data between studies or from different countries. Indeed the question has to be asked, 'What is being measured?'

1. Mair L H. Wear in dentistry-current terminology. J Dent 1992; 20: 140-144.

2. Gregg T, Mace $S$, West N X, Addy M. A study in vitro of the abrasive effect of the tongue on enamel and dentine by acid erosion. Caries Res 2004: 38: 557-560.

3. Last J M. A dictionary of epidemiology. 4th ed. Oxford University Press, 2001.

4. Ganss C, Klimek J, Lussi A. Accuracy and consistency of the visual diagnosis of exposed dentine on worn occlusal/incisal surfaces. Caries Res 2006; 40: 208-212.

5. O'Brien M. Children's dental health in the United Kingdom, 1993. London: HMSO, 1994.

6. Bardsley P F, Milosevic A, Taylor S. Epidemiological studies of tooth wear and dental erosion in 14-year-old children in North West England. Part 1 The relationship with water fluoridation and social deprivation. Br Dent J 2004; 197: 413-416.

7. Bartlett D, Ganss C, Lussi A. Basic Erosive Wear Examination (BEWE): a new scoring system for scientific and clinical needs. Clin Oral Investig 2008; 12 (Suppl 1): S65-S68.

8. Bartlett D. A proposed system for screening tooth wear. Br Dent J 2010; 208: 207-209.

9. Milosevic A, Young P J, Lennon M A. The prevalence of tooth wear in 14-year-old school children in Liverpool. Community Dent Health 1994; 11: 83-86.

10. Al-Dlaigan $Y H$, Shaw L, Smith A. Dental erosion in a group of British 14-year-old schoolchildren. Part I: Prevalence and influence of differing socioeconomic backgrounds. Br Dent J 2001; 190: 145-149.

11. Dugmore C R, Rock W P. The prevalence of tooth erosion in 12-year-old children. Br Dent J 2004; 196: $279-282$

12. Steele J, Walls A W G. Using partial recording to assess tooth wear in older adults. Community Dent Oral Epidemiol 2000; 28: 18-25.

13. Chadwick B L, White D A, Morris A J, Evans D, Pitts $\mathrm{N}$ B. Non-carious conditions in children in the UK, 2003. Br Dent J 2006; 200: 379-384.

14. Smith $B G N$, Knight J K. An index for measuring the wear of teeth. Br Dent J 1984; 156: 435-438.

15. Eccles J D. Dental erosion of non-industrial origin. A clinical survey and classification. J Prosthet Dent 1979; 42: 649-653.

16. Ganss C, Lussi A. Current erosion indices flawed or valid? Clin Oral Investig 2008: 12 (Suppl 1): S1-S3. 